\title{
MECÁNICA DE LOS DIAGRAMAS TERNARIOS: APLICACIÓN EN EL DIAGRAMA DE CLASIFICACIÓN DE LAS ROCAS ÍGNEAS DE STRECKEISEN
}

CASTAÑO, S.

Departamento de Ingeniería Geológica y Minera. Universidad de Castilla - La Mancha. 02006 Albacete.

\section{SUMMARY}

This paper develops the mechanics of the use of the ternary diagrams for the quantitative petrologic classifications and their application in one specific case: the Streckeisen's diagram for the igneous rocks.

\section{INTRODUCCIÓN}

Las clasificaciones petrológicas basadas en elementos cuantificables surgen como herramienta para lograr mayor precisión y objetividad en la determinación de ciertos grupos de rocas (Jung y Brousse 1959); a pesar de su finalidad original, cada vez se usan más en la enserianza de la geología a niveles medios (BUP). Su uso y comprensión no disminuye la importancia y valor didáctico de otras clasificaciones tradicionalmente empleadas, antes bien las complementan en aquellos aspectos en los cuales las características en las que se basan no son elementos fácilmente comprensibles para el alumno. Muchas de estas clasificaciones se concretan en la elaboración de diagramas gráficos de proporcionalidad entre diversas variables. De ellas existen numerosas aplicaciones: clasificaciones de rocas sedimentarias (Pettijohn 1957, Folk 1968), de rocas ígneas (Streckeisen 1965 y 1973), diagramas de tres componentes (AKF y ACF) o de estabilidad para rocas metamorficas (Winkler 1965, Portugal y Ferreira 1972) y los diagramas de $n$ variantes usados en petrogénesis.

La clasificación de Streckeisen para las rocas f́gneas es quizás el diagrama triangular más conocido a nivel docente, por aparecer en numerosos libros de geología de enseñanza media y COU. A pesar de su implantación (o quizás debido a ella), la bibliografía existente presupone el conocimiento del uso de tales diagramas y no suele entrar en la explicación de los mismos. Pocos autores (Turner y Verhoogen 1960, Bayly 1968, CorraIes Zarauza et al. 1977) introducen en sus obras alguna indicación sobre su mecánica. Estas clasificaciones resultan incomprensibles para los alumnos si antes no dominan el funcionamiento de los diagramas; su racionalidad y utilidad se pierde si no entienden su génesis.

\section{DIAGRAMAS BINARIOS}

Los diagramas binarios son la representación gráfica de las posibles combinaciones porcentuales entre dos variables, oscilando cada una de ellas entre el 0 y el $100 \%$ (Fig. 1). Consisten en una línea recta que presenta en cada extremo una variable; la proporción de la misma presente en la mezcla varía entre el $100 \%$ de la misma en su extremo y el $0 \%$ en el extremo correspondiente a la otra variable.

\section{Figura 1}

En el punto AA existe un $0 \%$ de la variable $\mathrm{B}$ y un $100 \%$ de la variable A. Conforme se avanza sobre la recta hacia la derecha, alejándonos de "A», el \% de la variable B aumenta hasta llegar al $100 \%$ y el \% de la variable A disminuye hasta el $0 \%$ sobre el punto «B». En el punto «B» hay un $0 \%$ de $\mathrm{A}$; conforme nos desplazamos sobre la recta del punto «B» al punto «A», el \% de $A$ va aumentando hasta llegar al $100 \%$.
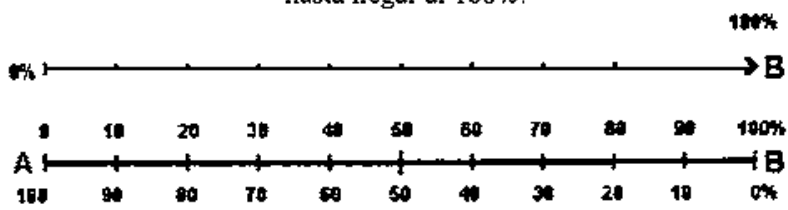

A $\rightarrow$ 


\section{DIAGRAMAS TERNARIOS}

Los diagramas ternarios o triangulares son la representación gráfica de las posibles relaciones o combinaciones entre tres elementos. Indican la distribución de tres variables o componentes diferentes y consisten en la unión de tres diagramas binarios en los que las variables se repiten dos a dos. Permiten trabajar con dos tipos de condiciones entre las variables: condiciones de proporción y condiciones de relación. Esto repercute en el tipo de líneas que configuran el gráfico.

La figura 2 muestra un diagrama ternario en el que cada vértice representa el $100 \%$ de la variable en él indicada y las bases opuestas el $0 \%$ de la misma.

Cada uno de los lados, considerados individualmente, constituye un diagrama binario. Los puntos situados en las líneas que configuran los lados del triángulo indican que en la composición total sólo hay dos de los tres componentes: aquéllos que son los vértices del diagrama binario al que pertenezca el punto.

\section{Figura 2}

Los puntos interiores del triángulo indican la mezcla de los tres componentes: A, B, y C.

Los puntos de la línea $x$ indican un $0 \%$ de $\mathrm{A}$. el $100 \%$ lo componen $B+C$.

Los puntos de la línea $y$ indican un $0 \%$ de $C$, el $100 \%$ lo forman $A+B$

Los puntos de la línea $z$ indican un $0 \%$ de $B$, e] $100 \%$ está compuesto por $\mathrm{A}+\mathrm{C}$.

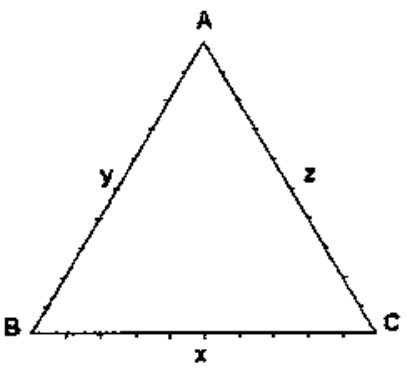

\section{Diagramas ternarios con líneas de proporcionalidad}

Las lineas en este diagrama están formadas por los puntos que configuran los lugares geométricos en los que el porcentaje de una variable se mantiene constante. Estas líneas, las podemos denominar «líneas de proporcionalidad». Cada una de ellas es en realidad un diagrama binario que reparte entre dos variables la proporción que resta tras atribuir al tercer componente un porcentaje fijo y determinado.

Para obtener una línea que represente un porcentaje determinado de uno de los elementos considerados ( A, B o C), se traza una paralela en el lado opuesto al vértice ocupado por dicho componente de forma tal que sus extremos sean los valores deseados. Estos valores vienen dados por los diagramas binarios que constituyen los lados que participan del componente analizado.
Figura 3

Las líneas de proporcionalidad son paralelas a uno de los lados y mantienen fija en todos sus puntos la proporción del elemento que ocupa eI vértice opuesto, independientemente de la relación entre las proporciones de los otros dos elementos.

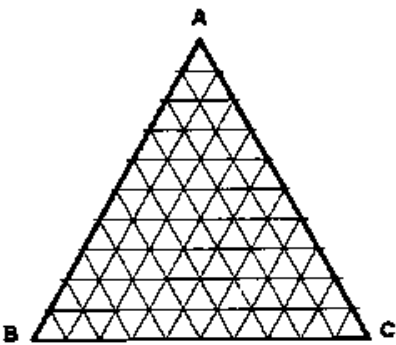

Figura 4

Los puntos de tas líneas de esta figura cumplen las siguientes condiciones:

Línea a: todos los puntos que la forman tienen el $80 \%$ de $\mathrm{A}$ y el $20 \%$ de $\mathrm{C}+\mathrm{B}$

Línea $b$ : todos los puntos tienen el $40 \%$ de $\mathrm{B}$ y el $60 \%$ restante de $\mathrm{A}+\mathrm{C}$

Línea $c$ : todos los puntos tienen el $50 \%$ de $\mathrm{C}$ el otro $50 \%$ es de $\mathrm{A}+\mathrm{B}$

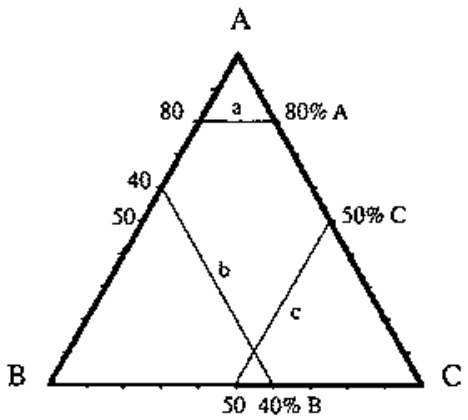

Es obvio que la concurrencia de diferentes condiciones a cumplir por los puntos del triángulo determinarán diferentes lugares geométricos que las cumplan. Estos pueden ser de tres tipos: puntos, líneas o zonas (figuras 5-7).

Figura 5

Caso 1: Punto.

Condiciones: $\begin{array}{lll}\text { 1) } \mathrm{A}=20 \% & \text { 2) } \mathrm{B}=60 \% & 3 \text { ) } \mathrm{C}=20 \%\end{array}$

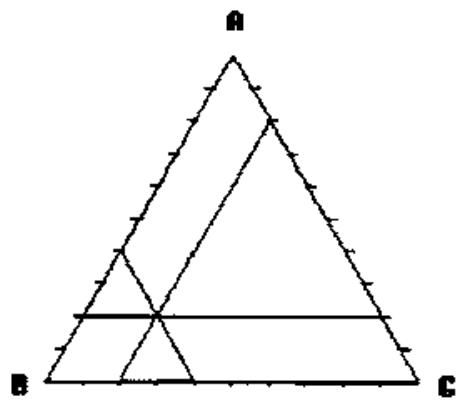


Figura 6

Caso 2: Línea

Condiciones: 1) $10 \%<\mathrm{A}<30 \%$ 2) $\mathrm{B}=30 \%$ 3) $40 \%<\mathrm{C}<60 \%$

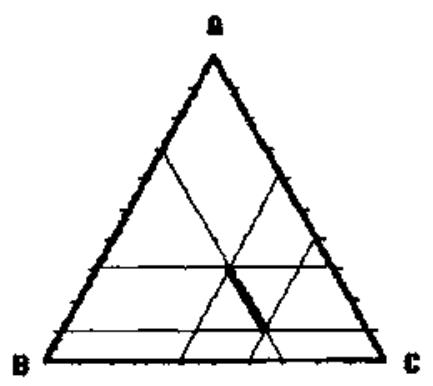

Figura 7

Caso 3: Zona

Condiciones: 1) $50 \%<\mathrm{A}<70 \%$ 2) $10 \%<\mathrm{B}<40 \%$

3) $10 \%<\mathrm{C}<20 \%$

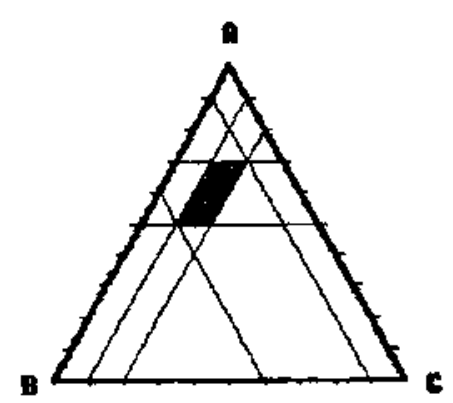

\section{Diagramas ternarios con líneas de relación}

Los lados de este diagrama son diagramas binarios, to mismo que en el diagrama ternario de proporción; sin embargo, las líneas interiores son los lugares geométricos en los que la proporción entre dos variables es siempre constante. Estas líneas las denominaremos «líneas de relación» (figura 8 ).

\section{Figura 8}

Las líneas interiores van siempre de un vértice al lado opuesto e indican la relación existente entre los porcentajes de las variables que forman ese fado, independientemente del valor de la variable que esté en el vértice.

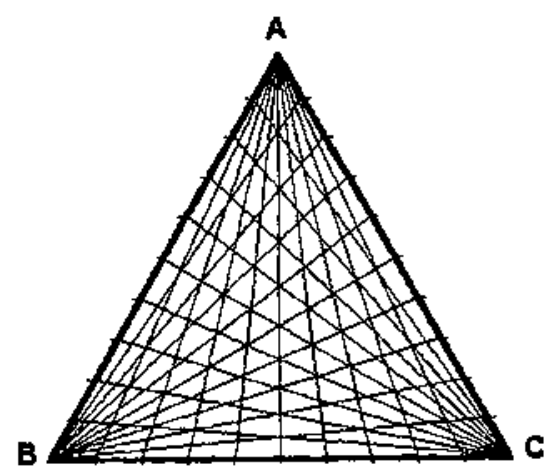

Figura 9

Los puntos de las líneas de esta figura cumplen las siguientes condiciones:

Línea $a$ : independientemente del porcentaje de A que exista, es decir, sobre un total de $\mathrm{B}+\mathrm{C}=100$, existe un $30 \%$ de $\mathrm{B}$ y un $70 \%$ de $C$, por Io que podemos establecer la relación: $\mathrm{B} / \mathrm{C}=30 / 70 \circ \mathrm{B} / \mathrm{C}=3 / 7$.

Línea $b$ : independientemente del porcentaje de $\mathrm{B}$ que exista, $\mathrm{A} / \mathrm{C}=80 / 20=8 / 2=4$.

Línea $c$ independientemente del porcentaje de $\mathrm{C}$ que exista, $\mathrm{A} / \mathrm{B}=60 / 40=6 / 4=3 / 2$.

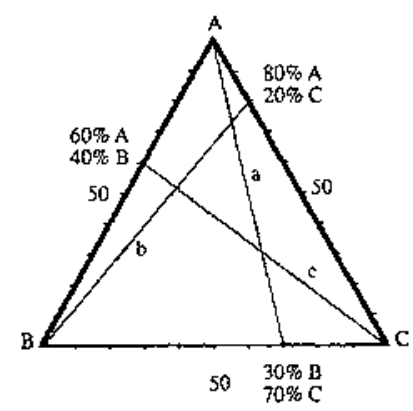

Como en el caso de los diagramas con líneas de proporción, diferentes condiciones determinan diferentes lugares geométricos, que también pueden ser de tres tipos: puntos, líneas o zonas (figuras 10-12).

Figura 10

Caso 1: Punto

Condiciones:

$\begin{array}{ll}\text { 1) } \mathrm{A} / \mathrm{B}=20 \% / 80 \%=1 / 4 & \text { 2) } \mathrm{B} / \mathrm{C}=50 \% / 50 \%=1\end{array}$

3) $\mathrm{C} / \mathrm{A}=80 \% / 20 \%=4$

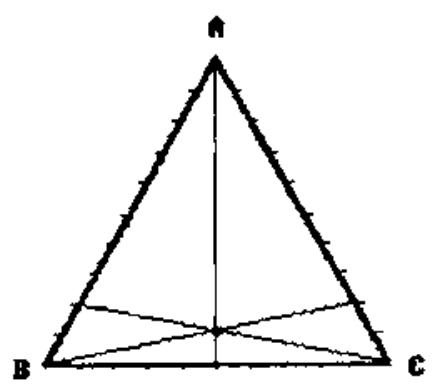

Figura 11

Caso 2: Línea

Condiciones:

1) $40 \% / 60 \%<\mathrm{A} / \mathrm{B}<70 \% / 30 \% \circ 2 / 3<\mathrm{A} / \mathrm{B}<7 / 3$

2) $\mathrm{B} / \mathrm{C}=70 \% / 30 \%=7 / 3$

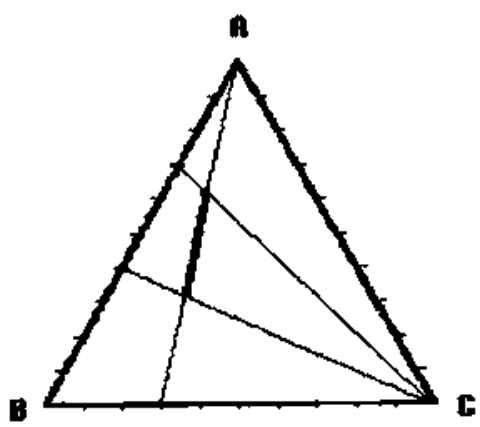

ENSEÑANZA DE LAS CIENCIAS, 1994, 12 (3) 
Figura 12

Caso 3: Zona

Condiciones:

i) $10 \% / 90 \%<\mathrm{B} / \mathrm{C}<40 \% / 60 \% \circ \mathrm{O} / \mathrm{S}<\mathrm{B} / \mathrm{C}<2 / 3$

2) $50 \% 50 \%<\mathrm{C} / \mathrm{A}<70 \% / 30 \%$ o $1<\mathrm{C} / \mathrm{A}<7 / 3$

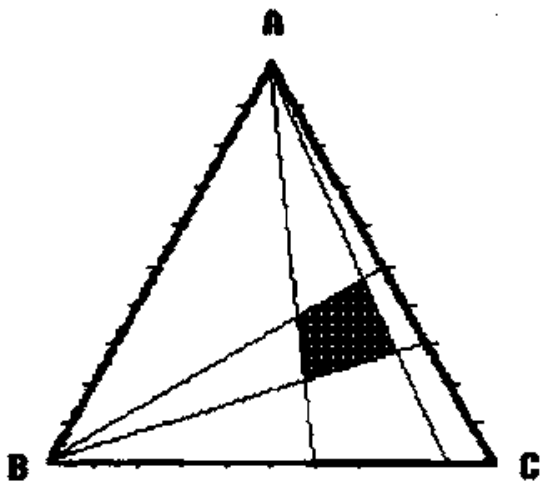

Figura 14
$A<65 \%$
$\mathrm{C}<50 \%$
$4>\mathrm{A} / \mathrm{B}>1$
$\mathrm{B} / \mathrm{C}<7 / 3$

Como se ve, son dos condiciones de proporcionalidad (\% de A y de $C$ ) y dos condiciones de relación (de $A / B$ y $B / C$ ). Todas ellas nos definen la zona punteada de la figura 14, único lugar geomécrico donde se cumplen las cuatro condiciones.

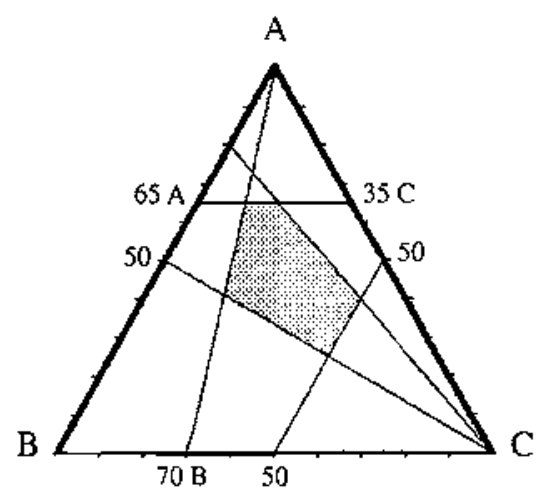

\section{CLASIFICACIÓN TRIANGULAR PARA LAS ROCAS IGNEAS}

Las clasificaciones de Streckeisen (1965), asumida por la IUGS como diagrama triangular para la clasificación de las rocas ígneas consiste en un doble diagrama ternario realizado en base a cuatro variables. Feldespato alcalino, plagioclasas, cuarzo y su extremo incompatible, los feldespatoides; del mismo quedan excluidas las rocas ultramáficas, con un porcentaje de ferromagnésicos superior al $90 \%$ en volumen (mafititas). Un precedente de la misma es la clasificación de Jung y Brousse (1959), basada en la medida y aplicación de los índices de saturación, feldespatos y coloración.

Figura 15

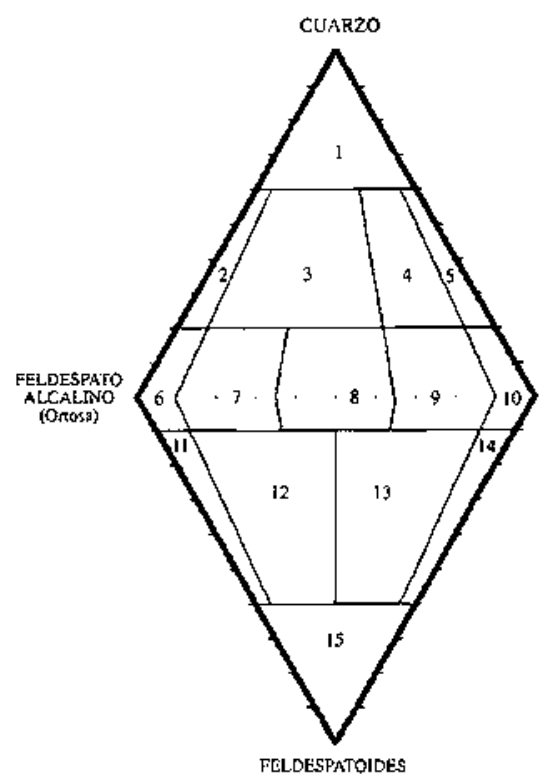

FELDESPATO (PLACHOCLASA) (Albirin-Anostia)
Las condiciones a cumplir por los puntos del triángulo pueden darse combinando condiciones de relación de proporción. Los diagramas aplicados conservan únicamente las líneas que delimitan zonas, facies o campos de estabilidad y usan aquuéllas que correspondan más exactamente a la condición que define ese límite. Cualquier tipo de proporción o relación entre tres variables nos permite definir un lugar geométrico (punto, línea o zona) cuyos puntos cumplen dichas condiciones; por ejemplo:
Figura 13

ENSEÑANZA DE LAS CIENCIAS, 1994, 12 (3) 
El índice de saturación,

$$
\mathrm{SAT}=\frac{\mathrm{Q}}{\mathrm{Q}+\mathrm{FatoS}} \times 100 \text { o SAT }=\frac{\text { foides }}{\text { foides }+ \text { Fatos }} \times 100
$$

puede aplicarse también en esta clasificación; se considera positivo para el diagrama del cuarzo y negativo para el de los feldespatoides. Resulta obvio que, conforme nos desplazamos en el diagrama vértice del cuarzo hacia el de los feldespatoides (independientemente del valor del índice feldespático), nos estamos desplazando en un sentido de disminución de los valores del índice SAT y de aumento de la basicidad de la roca.

\section{REFERENCIAS BIBLIOGRÁFICAS}

BAYLY, B., 1968. Introducción a la Petrologia. (Paraninfo: Madrid, 1971).

CORRALES ZARAUZA, I., ROSELL SANUY, I., SÁNCHEZ DE LA TORRE, L., VERA TORRES, J.A. y VILAS MINONDO, L., 1977. Estratigrafia. (Rueda: Madrid).

FOLK, R., 1968. Petrology of Sedimentary Rocks. (Hemphill's Austin: EEUU).

IUNG, J. y BROUSSE, R., 1959. Classification modale des Roches Eruptives. (Masson \& Cie: París).

PETTIJOHN,F.J., 1957. Sedimentary Rocks. (Harper \& Brothers: Nueva York).
Como se puede ver en la figura 15 , se trata de dos diagramas triangulares unidos por una base común que constituye una diagrama binario para ortosa y plagioclasas (que participan por igual de los dos triángulos), y dos vértices, el cuarzo y los feldespatoides (incompatibles entre sí). En él tienen cabida tanto las rocas plutónicas (asimilaremos este término a rocas de grano grueso, intrusivas o de consolidación lenta) como las rocas volcánicas (rocas de grano fino, extrusivas o de consolidación rápida).

En base a todo lo expuesto anteriormente y aplicando la mecánica de funcionamiento de los diagramas ternarios, podemos establecer las condiciones de relación y proporción que definen cada una de las zonas (tipos o familias de rocas) del diagrama del apéndice:

PORTUGAL, V, y FERREIRA, M.R., 1972. Rochas Metamorficas. (Gráfica de Coimbra: Portuga1).

STRECKEISEN, A, 1965. Die Klassifikation der Eruptivgesteine, Geologische rundschau, 55, pp. 478-491.

STRECKEISEN, A., 1973. Plutonic Rocks. Classification and nomenclature recommended by the IUGS Subcommission on the Systematics of Igneous Rocks, Geotimes, $18(10)$, pp. 26-30

TURNER, F.J. y VERHOOGEN, J., 1960. Petrologia ignea y metamórfica. (Omega: Barcelona, 1978).

WINKLER, H.G.F., 1965. Petrogenesis of Metamorphic Rocks. (Springer-Verlag Inc: Nueva York). 


\section{APÉNDICE}

Plutónicas

(Grano grueso)
Volcánicas

(Grano fino)

Riolitas alcalinas

Riolitas

Dacitas

Dacitas

Andesitas y basaltos cuarzosos

Traquitas alcalinas

Traquitas

Traquiandesita, latita

Andesita-latita

Basalto-latita

Basalto

Andesita

Fonolita

Fonolita tefrítica

Tefrita fonolítica

Basanita fonolítica

Basanita

Tefrita

Vulcanitas foidíticas 0

foiditas fonolíticas 0

feldespatoiditas extrusivas 